\title{
Phytoplankton biomass and production off the Baja California Peninsula: 1997-2016
}

\section{Biomasa y producción del fitoplancton frente a la península de Baja California: 1997-2016}

\author{
Martín E De la Cruz-Orozco ${ }^{1 \star}$, Eliana Gómez-Ocampo ${ }^{1}$, Luis E Miranda-Bojórquez ${ }^{1}$, Jushiro \\ Cepeda-Morales ${ }^{2}$, Reginaldo Durazo ${ }^{3}$, Bertha E Lavaniegos ${ }^{1}$, Teresa L Espinosa-Carreón ${ }^{4}$, Ramón \\ Sosa-Ávalos ${ }^{5}$, Elsa Aguirre-Hernández ${ }^{1}$, Gilberto Gaxiola-Castro ${ }^{1 \dagger}$ \\ ${ }^{1}$ División de Oceanología, Centro de Investigación Científica y de Educación Superior de Ensenada, Carretera \\ Ensenada-Tijuana, no. 3918, Zona Playitas, CP 22860, Ensenada, Baja California, México. \\ 2 Universidad Autónoma de Nayarit, Ciudad de la Cultura "Amado Nervo", CP 63155, Tepic, Nayarit, México. \\ 3 Facultad de Ciencias Marinas, Universidad Autónoma de Baja California, Carretera Ensenada-Tijuana, \\ no. 3918, Zona Playitas, Ensenada, CP 22860, Baja California, México. \\ ${ }^{4}$ Centro Interdisciplinario de Investigación para el Desarrollo Integral Regional (CIIDIR), Unidad Sinaloa- \\ IPN, Blvd. Juan de Dios Bátiz Paredes, no. 250, colonia San Joachín, CP 81101, Guasave, Sinaloa, México. \\ 5 Facultad de Ciencias Marinas, Universidad de Colima, Carretera Manzanillo-Cihuatlán km. 20, CP 29860, \\ Manzanillo, Colima, México. \\ * Corresponding author. E-mail: delacruz@cicese.mx
}

\begin{abstract}
Between 1997 and 2016, the Investigaciones Mexicanas de la Corriente de California (IMECOCAL, Mexican Investigations of the California Current) program conducted measurements of chlorophyll $a$ and phytoplankton production off Baja California. Here, we summarize the main results obtained during the survey period. Long-term means indicated that high productivity occurred along the coastal zone where upwelling occurs, and in a semi-permanent cyclonic gyre off Punta Eugenia. At the seasonal scale, the variability of integrated primary production showed differences with respect to integrated chlorophyll $a$ variability. Integrated chlorophyll $a$ exhibited maximum values during the coastal upwelling season in spring and summer, while minimum values occurred during the inflow of tropical-subtropical waters south of Punta Eugenia in autumn and winter. In contrast, the highest values of integrated primary production occurred in spring and summer to the north of Punta Eugenia, whereas to the south of this location, the highest values occurred in winter and autumn. This seasonal variability was associated with phytoplankton size and maximum photosynthetic rates. At the interannual scale, low productivity was associated with increased stratification during the subarctic water intrusion events and the "Blob" and El Niño events in 2015-2016. Productivity time-series were in agreement with the seasonal variability of the north Pacific Gyre Oscillation index. Given that phytoplankton is the first level of the marine food web, this study contributes to the understanding of regional variations in higher trophic levels at the seasonal and interannual scales.
\end{abstract}

Key words: integrated chlorophyll $a$, integrated primary production, California Current, climatic variations, North Pacific Gyre Oscillation.

RESUMEN. Entre 1997 y 2016, en el programa de Investigaciones Mexicanas de la Corriente de California (IMECOCAL) se realizaron mediciones de clorofila $a$ y producción del fitoplancton frente a la península de Baja California. En este trabajo se resumen los resultados del periodo de observaciones. Se encontró que la productividad promedio fue alta en zonas de surgencias costeras, y en el giro ciclónico semipermanente frente a punta Eugenia. A escala estacional, la variación de la producción primaria fue diferente a la de la clorofila $a$ integrada. La clorofila $a$ integrada presentó máximos durante la época de surgencias costeras en primavera y verano, y mínimos durante la influencia de agua cálida de origen tropical-subtropical en la zona al sur de punta Eugenia en otoño e invierno. Los mayores valores de producción primaria integrada ocurrieron en primavera y verano al norte de punta Eugenia y en invierno y otoño al sur de esta localidad. Esta variabilidad se relacionó con la presencia de fitoplancton de ciertos tamaños y sus tasas fotosintéticas máximas características. A escala interanual, la menor productividad estuvo asociada con la mayor estratificación durante los eventos de intrusión de agua subártica, y los eventos el "Blob" y El Niño entre 2015 y 2016. Los cambios en la productividad observados concuerdan con la variación temporal del índice de la Oscilación del Giro del Pacifico Norte. La variabilidad observada en ambas escalas contribuye a entender la variación regional en niveles tróficos superiores, dado que el fitoplancton es el principal componente de la cadena trófica marina.

Palabras clave: clorofila $a$ integrada, producción primaria integrada, corriente de California, variabilidad climática, Oscilación del Giro del Pacifico Norte. 


\section{INTRODUCTION}

The ocean surface layer (0-100 m) off the Baja California Peninsula is influenced by the southern end of the California Current (CC). This region is considered a transition zone (Roden 1971) due to the confluence of water masses with different physicochemical properties: Subarctic Water that is transported southward by the CC, Tropical Surface Water, and Subtropical Surface Water that flows from the areas south and southwest of the peninsula (Hickey 1998, Durazo 2015). Biological diversity and the physicochemical variability in the region are modulated by the interactions and boundaries between water masses and their spatiotemporal variability (Bograd and Lynn 2003, Gaxiola-Castro et al. 2008). For example, at the seasonal scale, the $\mathrm{CC}$ is more intense and influences the entire region off Baja California during spring, whereas during autumn-winter the weakening of this current allows waters of tropical and subtropical origin to enter the region. This variability divides the peninsula into 2 regions: one with subarctic characteristics to the north of Punta Eugenia and one with warmer conditions to the south of Punta Eugenia (Durazo 2009, 2015). This seasonal change in environmental conditions is reflected in the variations of phytoplankton biomass and production and of zooplankton groups (Lavaniegos and Ohman 2003, Gómez-Ocampo et al. 2017a).

Phytoplankton biomass and production off Baja California have different spatiotemporal scales. High chlorophyll $a$ $\left(\mathrm{Chla}, \mathrm{mg} \cdot \mathrm{m}^{-3}\right)$ values were reported for spring as a result of intensified wind stress, which causes upwelling of nutrient-rich water along the peninsula (Durazo 2015). The mesoscale eddies, fronts, and meanders that occur as a result of friction between currents have caused changes in biomass and phytoplankton groups (Espinosa-Carreon et al. 2004, 2012). At the interannual scale, events such as the subarctic water intrusion in 2002-2006, the Blob, and the 2015-2016 El Niño caused phytoplankton biomass and production to decline (Espinosa-Carreón et al. 2015, Gómez-Ocampo et al. 2017b). Therefore, understanding changes in phytoplankton biomass and production in the region off Baja California at these scales and their possible implications on the food web is essential to determine oceanic carbon flux and changes in the upper trophic levels, such as zooplankton, small pelagic fish, and/or fish larvae.

In the present analysis all data collected between 1997 and 2016 by the IMECOCAL (Spanish acronym for Mexican Investigations of the California Current) program are used. The analysis will allow a more precise evaluation of average conditions and seasonal variability in phytoplankton biomass and production in the region. For this reason, the aim of this article is to characterize the spatiotemporal variability in the water column using Chla and primary production data obtained during the IMECOCAL oceanographic campaigns. The results are used to discuss the main seasonal and interanual processes that impact the pelagic ecosystem in this region.

\section{INTRODUCCIÓN}

El océano superficial $(0-100 \mathrm{~m})$ frente a la península de Baja California se encuentra influenciado por el límite sur de la corriente de California (CC). Esta región es considerada como una zona de transición (Roden 1971) debido a la confluencia de varias masas de agua con diferentes propiedades fisicoquímicas: Agua Subártica transportada hacia el sur por la CC, Agua Tropical Superficial y Agua Subtropical Superficial que proviene de la zona frente al sur y suroeste de la península (Hickey 1998, Durazo 2015). Las interacciones y los límites entre las masas de agua que allí confluyen, y su variación espaciotemporal, modulan la diversidad biológica y la variabilidad física y química de la región (Bograd y Lynn 2003, Gaxiola-Castro et al. 2008). Por ejemplo, en la escala estacional, se ha observado que durante la primavera la CC es más intensa y tiene influencia en toda la región frente a Baja California, mientras que en otoño e invierno su debilitamiento permite la entrada de aguas de origen tropical y subtropical. Esta variabilidad divide la península en 2 provincias: una al norte de punta Eugenia con carácter subártico y otra al sur de punta Eugenia con condiciones más cálidas (Durazo 2009, 2015). Este cambio estacional en las condiciones ambientales se ha visto reflejado en variaciones de la biomasa y producción del fitoplancton, así como en grupos de zooplancton (Lavaniegos y Ohman 2003, Gómez-Ocampo et al. 2017b).

La biomasa y la producción del fitoplancton frente a Baja California se han caracterizado por presentar variaciones en diferentes escalas espaciotemporales. Se han reportado valores altos de clorofila $a\left(\mathrm{Cl} a, \mathrm{mg} \cdot \mathrm{m}^{-3}\right)$ durante la primavera como resultado de la intensificación del esfuerzo del viento, que trae como consecuencia el afloramiento de aguas ricas en nutrientes a lo largo de la península de Baja California (Durazo 2015). En la mesoescala, los remolinos, frentes y meandros que ocurren por la fricción de las corrientes han generado cambios en la biomasa y grupos del fitoplancton (Espinosa-Carreón et al. 2004, 2012). En la escala interanual, eventos como la intrusión de agua subártica de 2002 a 2006, el "Blob" y El Niño de 2015-2016 generaron la reducción en la biomasa y la producción del fitoplancton (Espinosa-Carreón et al. 2015, Gómez-Ocampo et al. 2017b). Por lo tanto, entender los cambios en la biomasa y la producción del fitoplancton a estas escalas en la región frente a Baja California y su posible impacto en la trama trófica es esencial para determinar los flujos del carbono en el océano y, con ello, los cambios en niveles tróficos superiores, tales como el zooplancton, los pelágicos menores y/o las larvas de peces.

En el presente análisis se considera el total de datos recolectados entre 1997 y 2016 (20 años) por el programa Investigaciones Mexicanas de la Corriente de California (IMECOCAL). El análisis permitirá evaluar de forma más aproximada las condiciones promedio y la variación estacional de la producción y la biomasa del fitoplancton en la región. Por tal motivo, el objetivo del presente artículo es caracterizar la variación espaciotemporal de la productividad 
De la Cruz-Orozco et al.: Phytoplankton biomass and production off Baja California

\section{MATERIALS AND METHOdS}

The data used for this study were collected during approximately 60 cruises that were carried out along the station grid that covers the study area (Fig. 1). Water samples for the Chla $\left(\mathrm{mg} \cdot \mathrm{m}^{-3}\right)$ analysis were collected with 5 -L Niskin bottles (General Oceanics) at standard depths $(0,10,20,50,100$, 150 , and $200 \mathrm{~m}$ ). Each sample was analyzed with the fluorescence method (Yentsch and Menzel 1963, Holm-Hansen et al. 1965). Primary production (PP, $\mathrm{mg} \mathrm{C} \cdot \mathrm{m}^{-3} \cdot \mathrm{h}^{-1}$ ) was determined in situ with the ${ }^{14} \mathrm{C}$ method (Steeman-Nielsen 1952) in samples collected between 11:00 and 13:00 local time at 6 irradiance levels $(100 \%, 50 \%, 30 \%, 20 \%, 10 \%$, and $1 \%$ surface irradiance) and incubated for $\sim 2 \mathrm{~h}$ in the water column. The results of both biological variables were vertically integrated using the trapezoidal rule to estimate integrated Chla $\left(\mathrm{Chl}_{\text {int }}\right.$, $\mathrm{mg} \cdot \mathrm{m}^{-2}$ ) from the surface to $100 \mathrm{~m}$ depth and integrated PP $\left(\mathrm{PP}_{\text {int }}, \mathrm{mg} \mathrm{C} \cdot \mathrm{m}^{-2} \cdot \mathrm{h}^{-1}\right)$ from the surface to the euphotic zone depth ( $1 \%$ surface irradiance).

In order to represent seasonal variation of productivity in the water column, seasonal composites (because for some seasons there was only one datum) of Chla $a_{\text {int }}$ and $\mathrm{PP}_{\text {int }}$ at the IMECOCAL sampling stations were calculated for winter (December, January, and February), spring (March, April, and May), summer (June, July, and August), and autumn (September, October, and November). Interannual variations were analyzed using the time series of the $\mathrm{Chla}_{\text {int }}$ and $\mathrm{PP}_{\text {int }}$ anomalies, which were defined as the observed value minus the average value calculated from 1997 to 2016. These variations were associated with 3 indexes representative of the interannual variability in the Pacific Ocean: the Pacific Decadal Oscillation (PDO; Mantua et al. [1997], http://jisao. washington.edu/pdo/PDO.latest), the Multivariate El Niño/ Southern Oscillation (ENSO) Index (MEI, https://www.esrl. noaa.gov/psd/enso/mei/rank.html), and the North Pacific Gyre Oscillation index (NPGO, Di Lorenzo et al. [2008]).

\section{Results}

\section{Average conditions}

Productivity in the water column was represented by integrated Chla and PP values (Fig. 2a, b). The highest Chla $a_{\text {int }}$ values $\left(\sim 150 \mathrm{mg} \cdot \mathrm{m}^{-2}\right)$ were observed along the coastal zone of the study area, between Vizcaíno Bay and Punta Baja $\left(28^{\circ} \mathrm{N}-30^{\circ} \mathrm{N}\right.$, Fig. 2a). The oceanic zone shows the lowest biomass values $\left(<50 \mathrm{mg} \cdot \mathrm{m}^{-2}\right)$ in the water column. However, a site with slightly higher values $\left(\sim 70 \mathrm{mg} \cdot \mathrm{m}^{-2}\right)$ than the ones observed for the oceanic zone is detected off Punta Eugenia $\left(28^{\circ} \mathrm{N}\right)$. The highest $\mathrm{PP}_{\text {int }}$ values $\left(150 \mathrm{mg} \mathrm{C} \cdot \mathrm{m}^{-2} \cdot \mathrm{h}^{-1}\right.$, Fig. $\left.2 \mathrm{~b}\right)$ in the region off Baja California occurred to the south and to the north of Punta Eugenia $\left(28^{\circ} \mathrm{N}\right)$, and to a lesser degree in the coastal zone $\left(\sim 100 \mathrm{mg} \mathrm{C} \cdot \mathrm{m}^{-2} \cdot \mathrm{h}^{-1}\right)$. Average $\mathrm{PP}_{\text {int }}$ values were lower $\left(<50 \mathrm{mg} \mathrm{C} \cdot \mathrm{m}^{-2} \cdot \mathrm{h}^{-1}\right)$ in the oceanic zone, except at one high productivity site off Punta Eugenia $\left(\sim 125 \mathrm{mg} \mathrm{C} \cdot \mathrm{m}^{-2} \cdot \mathrm{h}^{-1}\right)$. de la columna de agua a partir de la información de Cla y producción primaria, obtenidas en las campañas oceanográficas de IMECOCAL. Los resultados se utilizan para discutir los principales procesos en la escala estacional y en la escala interanual que impactan al ecosistema pelágico de esta región.

\section{MATERIALES Y MÉTODOS}

Se utilizó la información generada de aproximadamente 60 cruceros realizados a lo largo de la malla de estaciones que cubre la zona de estudio (Fig. 1). Las muestras de agua para el análisis de $\mathrm{Cla}\left(\mathrm{mg} \cdot \mathrm{m}^{-3}\right)$ se recolectaron a profundidades estándar de 0, 10, 20, 50, 100, 150 y $200 \mathrm{~m}$ utilizando botellas muestradoras Niskin de $5 \mathrm{~L}$ (General Oceanics). Cada muestra fue analizada con el método fluorimétrico (Yentsch y Menzel 1963, Holm-Hansen et al. 1965). La producción primaria (PP, $\mathrm{mg} \mathrm{C} \cdot \mathrm{m}^{-3} \cdot \mathrm{h}^{-1}$ ) fue determinada in situ con el método del ${ }^{14} \mathrm{C}$ (Steeman-Nielsen 1952) en muestras recolectadas entre las 11:00-13:00 (hora local) a 6 niveles de irradiancia (100\%, $50 \%, 30 \%, 20 \%, 10 \%$ y $1 \%$ de la irradiancia superficial) e incubadas por $\sim 2 \mathrm{~h}$ en la columna de agua. Los resultados de ambas variables biológicas fueron integrados verticalmente usando la regla trapezoidal para obtener la clorofila integrada $\left(\mathrm{Cl} a_{\mathrm{int}}, \mathrm{mg} \cdot \mathrm{m}^{-2}\right)$ desde la superficie hasta $100 \mathrm{~m}$ de profundidad, y la producción primaria integrada $\left(\mathrm{PP}_{\mathrm{int}}, \mathrm{mg} \mathrm{C} \cdot \mathrm{m}^{-2} \cdot \mathrm{h}^{-1}\right)$ desde la superficie hasta la profundidad de la zona eufótica ( $1 \%$ de la irradiancia superficial).

Con el fin de representar la variación estacional de la productividad de la columna de agua, se calcularon los compuestos estacionales (porque en algunas estaciones solo había un dato) de $\mathrm{Cl}_{\text {int }}$ y $\mathrm{PP}_{\text {int }}$ en las estaciones de muestreo del IMECOCAL para invierno (diciembre, enero y febrero), primavera (marzo, abril y mayo), verano (junio, julio y agosto) y otoño (septiembre, octubre y noviembre). Las variaciones interanuales se analizaron a partir de las series de tiempo de las anomalías de $\mathrm{Cla}_{\text {int }}$ y $\mathrm{PP}_{\text {int }}$, las cuales se definieron como el valor observado menos el promedio calculado desde 1997 hasta 2016. Estas variaciones se relacionaron con 3 índices representativos de la variabilidad interanual en el océano Pacífico: el índice de la Oscilación Decadal del Pacífico (PDO; Mantua et al. [1997], http://jisao.washington.edu/pdo/PDO.latest), el Índice multivariado de El Niño/Oscilación del Sur (MEI, por sus siglas en inglés; https://www.esrl.noaa.gov/psd/enso/mei/rank.html) y el índice que representa la Oscilación del Giro del Pacifico Norte (NPGO, Di Lorenzo et al. [2008]).

\section{RESUltados}

\section{Condiciones medias}

La productividad de la columna de agua se ve representada a través de los valores integrados de Cla y PP (Fig. 2a, b). Los mayores valores de $\mathrm{Cl}_{\text {int }}\left(\sim 150 \mathrm{mg} \cdot \mathrm{m}^{-2}\right)$ se observaron a lo largo de la zona costera del área de estudio, entre bahía de Vizcaíno y punta Baja $\left(28^{\circ} \mathrm{N}-30^{\circ} \mathrm{N}\right.$, Fig. 2a). La zona oceánica 


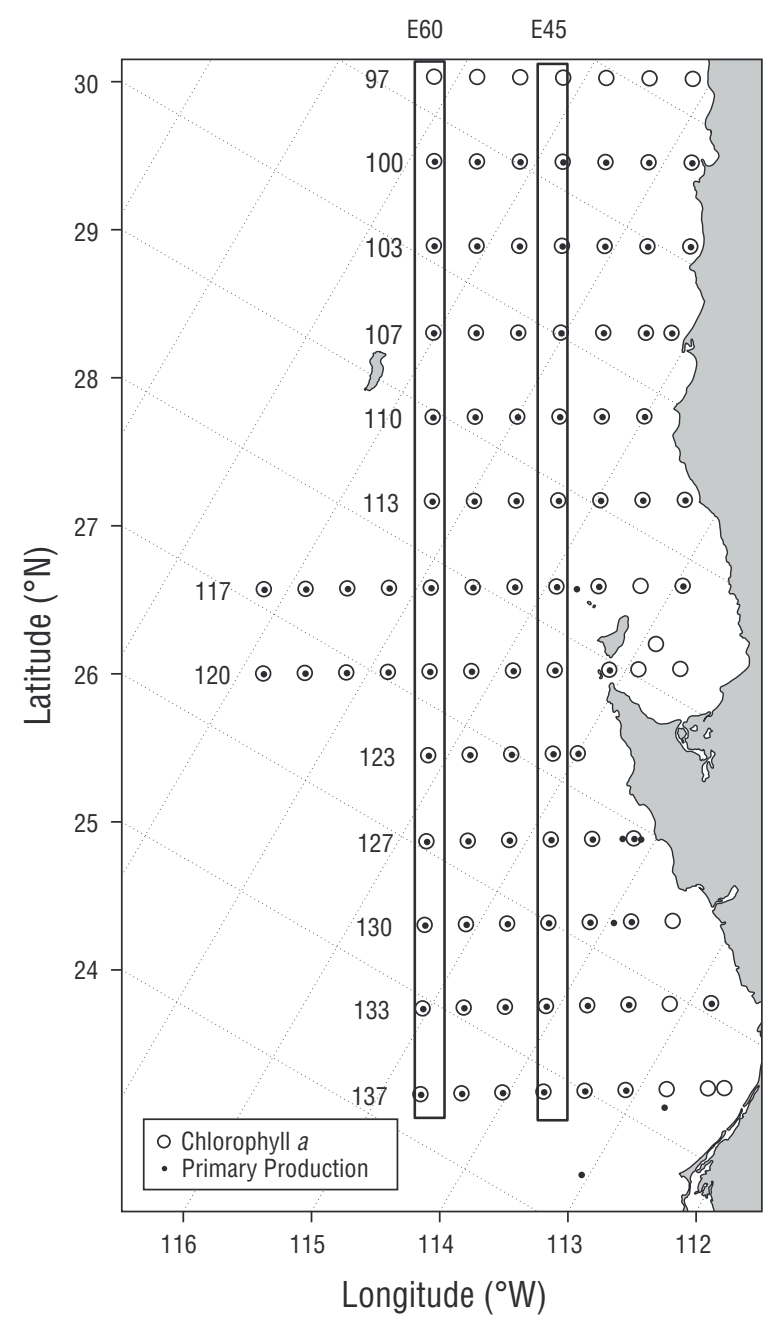

Figure 1. Hydrographic lines and stations of the IMECOCAL program where chlorophyll $a(\circ)$ and primary production $(\bullet)$ were measured. Boxes indicate the transects of stations 45 (E45) and stations 60 (E60), representative of transition and oceanic conditions, respectively.

Figura 1. Líneas hidrográficas y estaciones del programa IMECOCAL donde se midieron la clorofila $a(0)$ y la produccion primaria $(\bullet)$. Los rectángulos indican los transectos de las estaciones 45 (E45) y las estaciones 60 (E60), representativas de condiciones transicionales y oceánicas, respectivamente.

Average Chla values across stations 45 of each transect (E45) are highest at $20 \mathrm{~m}$ depth on line $117\left(\sim 1.3 \mathrm{mg} \cdot \mathrm{m}^{-3}\right)$, which is located off Vizcaíno Bay $\left(28^{\circ} \mathrm{N}\right)$. The Chla maximum $\left(\sim 0.8 \mathrm{mg} \cdot \mathrm{m}^{-3}\right)$ was generally located at $50 \mathrm{~m}$ depth. Likewise, on stations 60 of each transect (E60) (Fig. 2d) the highest Chla value $\left(\sim 0.9 \mathrm{mg} \cdot \mathrm{m}^{-3}\right)$ is observed at $50 \mathrm{~m}$ depth on line 117 . In addition, the formation of a boundary or a division between the northern (97-123) and southern (127-137) lines is observed. This division occurrs around lines 117 and 120, and separates the water column into 2 zones, a mesotrophic $\left(0.2 \mathrm{mg} \cdot \mathrm{m}^{-3}<\right.$ Chla $\left.<1 \mathrm{mg} \cdot \mathrm{m}^{-3}\right)$ zone in the northern portion and an oligotrophic $\left(\right.$ Chla $\left.<0.2 \mathrm{mg} \cdot \mathrm{m}^{-3}\right)$ zone in the southern portion, according to the classification proposed by Kahru and Mitchell (2002). presentó los valores más bajos de biomasa $\left(<50 \mathrm{mg} \cdot \mathrm{m}^{-2}\right)$ en la columna de agua. Sin embargo, frente a punta Eugenia $\left(28^{\circ} \mathrm{N}\right)$ se destaca un punto donde los valores de $\mathrm{Cla}_{\text {int }}$ son ligeramente más altos $\left(\sim 70 \mathrm{mg} \cdot \mathrm{m}^{-2}\right)$ que los observados en la zona oceánica. Los valores más altos de $\mathrm{PP}_{\text {int }}\left(150 \mathrm{mg} \mathrm{C} \cdot \mathrm{m}^{-2} \cdot \mathrm{h}^{-1}\right.$, Fig. $\left.2 \mathrm{~b}\right)$ en la región frente a Baja California ocurrieron al sur y al norte de punta Eugenia $\left(28^{\circ} \mathrm{N}\right)$, y en menor medida en la zona costera $\left(\sim 100 \mathrm{mg} \mathrm{C} \cdot \mathrm{m}^{-2} \cdot \mathrm{h}^{-1}\right)$. En la zona oceánica los valores promedio de $\mathrm{PP}_{\text {int }}$ fueron más bajos $\left(<50 \mathrm{mg} \mathrm{C} \cdot \mathrm{m}^{-2} \cdot \mathrm{h}^{-1}\right)$, excepto en un punto de alta productividad frente a punta Eugenia $(\sim 125 \mathrm{mg}$ $\left.\mathrm{C} \cdot \mathrm{m}^{-2} \cdot \mathrm{h}^{-1}\right)$. El promedio de Cla a lo largo de las estaciones 45 de cada transecto (E45) muestra el valor más alto a $20 \mathrm{~m}$ de profundidad en la línea $117\left(\sim 1.3 \mathrm{mg} \cdot \mathrm{m}^{-3}\right)$, la cual se encuentra ubicada frente a bahía de Vizcaíno $\left(28^{\circ} \mathrm{N}\right)$. En general, en el resto del transecto se observa el máximo de Cla $\left(\sim 0.8 \mathrm{mg} \cdot \mathrm{m}^{-3}\right)$ a $50 \mathrm{~m}$ de profundidad. Similarmente, en las estaciones 60 de cada transecto (E60) (Fig. 2d) se observa el valor más alto de Cla en la línea $117\left(\sim 0.9 \mathrm{mg} \cdot \mathrm{m}^{-3}\right)$ a $50 \mathrm{~m}$ de profundidad. También se puede observar la formación de una frontera o división entre las líneas norteñas (97 a 123) y las líneas sureñas (127 a 137). Esta división ocurre alrededor de las líneas 117 y 120 , y separa la columna de agua en 2 zonas, una mesotrófica $\left(0.2 \mathrm{mg} \cdot \mathrm{m}^{-3}<\mathrm{Cl} a<1 \mathrm{mG} \cdot \mathrm{m}^{-3}\right)$ en la porción norteña y otra oligotrófica $\left(\mathrm{Cla}<0.2 \mathrm{mg} \cdot \mathrm{m}^{-3}\right)$ en la porción sureña, según la clasificación propuesta por Kahru y Mitchell (2002).

\section{Variación estacional de la biomasa y la producción del fitoplancton}

Los promedios estacionales de la biomasa y de la producción de fitoplancton estimados para la zona IMECOCAL (Figs. 3, 4) mostraron que los valores más altos de la biomasa del fitoplancton integrados en la columna de agua ocurrieron durante invierno y primavera a lo largo de la zona costera y en algunas partes de la zona oceánica (Fig. 3). En invierno, sobresalió un lugar de alta $\mathrm{Cl}_{\text {int }}\left(\sim 300 \mathrm{mg} \cdot \mathrm{m}^{-2}\right)$ frente a punta Eugenia. En primavera, los valores de $\mathrm{Cl} a_{\text {int }}$ más altos $\left(\sim 300 \mathrm{mg} \cdot \mathrm{m}^{-2}\right)$ ocurrieron al norte del área de estudio. En verano y otoño, los valores de biomasa integrada fueron similares en la zona costera y la zona oceánica $\left(<100 \mathrm{mg} \cdot \mathrm{m}^{-2}\right)$. La $\mathrm{PP}_{\text {int }}$ presentó una mayor variación estacional en el área de estudio (Fig. 4) que la mostrada por la $\mathrm{Cla}_{\text {int }}$. En invierno, los valores más altos $\mathrm{PP}_{\text {int }}$ $\left(\sim 150 \mathrm{mg} \mathrm{C} \cdot \mathrm{m}^{-2} \cdot \mathrm{h}^{-1}\right)$ se presentaron al sur de punta Eugenia $\left(28^{\circ} \mathrm{N}\right)$. Durante la primavera, la región al norte de punta Eugenia mostró los máximos de $\mathrm{PP}_{\text {int }}\left(\sim 150 \mathrm{mg} \mathrm{C} \cdot \mathrm{m}^{-2} \cdot \mathrm{h}^{-1}\right)$. En verano, los valores más altos de $\mathrm{PP}_{\text {int }}\left(\sim 150 \mathrm{mg} \mathrm{C} \cdot \mathrm{m}^{-2} \cdot \mathrm{h}^{-1}\right)$ ocurrieron en bahía de Vizcaíno, mientras que en la región sur y frente a punta Eugenia ocurrieron en otoño.

\section{Anomalías en la biomasa y la producción y del fitoplancton (1997-2016)}

En los 20 años de mediciones de IMECOCAL se observaron variaciones interanuales notables en la biomasa y la producción del fitoplancton integradas en la columna de agua. 


\section{Seasonal variations of phytoplankton biomass and production}

The estimated phytoplankton biomass and production seasonal averages estimated for the IMECOCAL area (Figs. 3, 4) showed that the highest values of phytoplankton biomass integrated in the water column occurred during winter and spring along the coastal zone and in some parts of the oceanic zone (Fig. 3). In winter a site with outstandingly high Chla $a_{\text {int }}$ values $\left(\sim 300 \mathrm{mg} \cdot \mathrm{m}^{-2}\right)$ was observed off Punta Eugenia. In spring the highest Chla $a_{\text {int }}$ values $\left(\sim 300 \mathrm{mg} \cdot \mathrm{m}^{-2}\right)$ occurred to the north of the study area. During summer and autumn, integrated biomass values were similar in the coastal and oceanic zones $\left(<100 \mathrm{mg} \cdot \mathrm{m}^{-2}\right)$. The seasonal variation in the study area was higher for $\mathrm{PP}_{\text {int }}$ (Fig. 4) than for Chl $a_{\text {int }}$. In winter the highest $\mathrm{PP}_{\text {int }}$ values $\left(\sim 150 \mathrm{mg} \mathrm{C} \cdot \mathrm{m}^{-2} \cdot \mathrm{h}^{-1}\right)$ were
Durante varios periodos, ocurrió el declive en la biomasa del fitoplancton: El Niño de 1998, la intrusión de agua subártica del 2002 al 2007, el Blob y El Niño de 2015-2016. Las mayores disminuciones en la $\mathrm{Cla}_{\text {int }}$ se observaron durante la intrusión de agua Subártica (aproximadamente $-30 \mathrm{mg} \cdot \mathrm{m}^{-2}$ ) y durante los periodos de El Niño (aproximadamente $-20 \mathrm{mg} \cdot \mathrm{m}^{-2}$ ). En contraste, la ocurrencia de los eventos de La Niña de 1999, 2008 y 2010, y el incremento en la intensidad en las surgencias durante 2002 generaron un aumento en la biomasa del fitoplancton de hasta $90 \mathrm{mg} \cdot \mathrm{m}^{-2}$ (Fig. 5a). A diferencia de la biomasa, la producción del fitoplancton (Fig. 5b) no exhibió una respuesta tan clara a eventos interanuales. Sin embargo, se observaron incrementos de $\mathrm{PP}_{\text {int }}$ notables durante los eventos de La Niña 1999 y 2008, a finales del 2014 durante la transición de la ocurrencia del Blob y El Niño de 2015-2016, y a finales de 2016 durante el periodo neutral
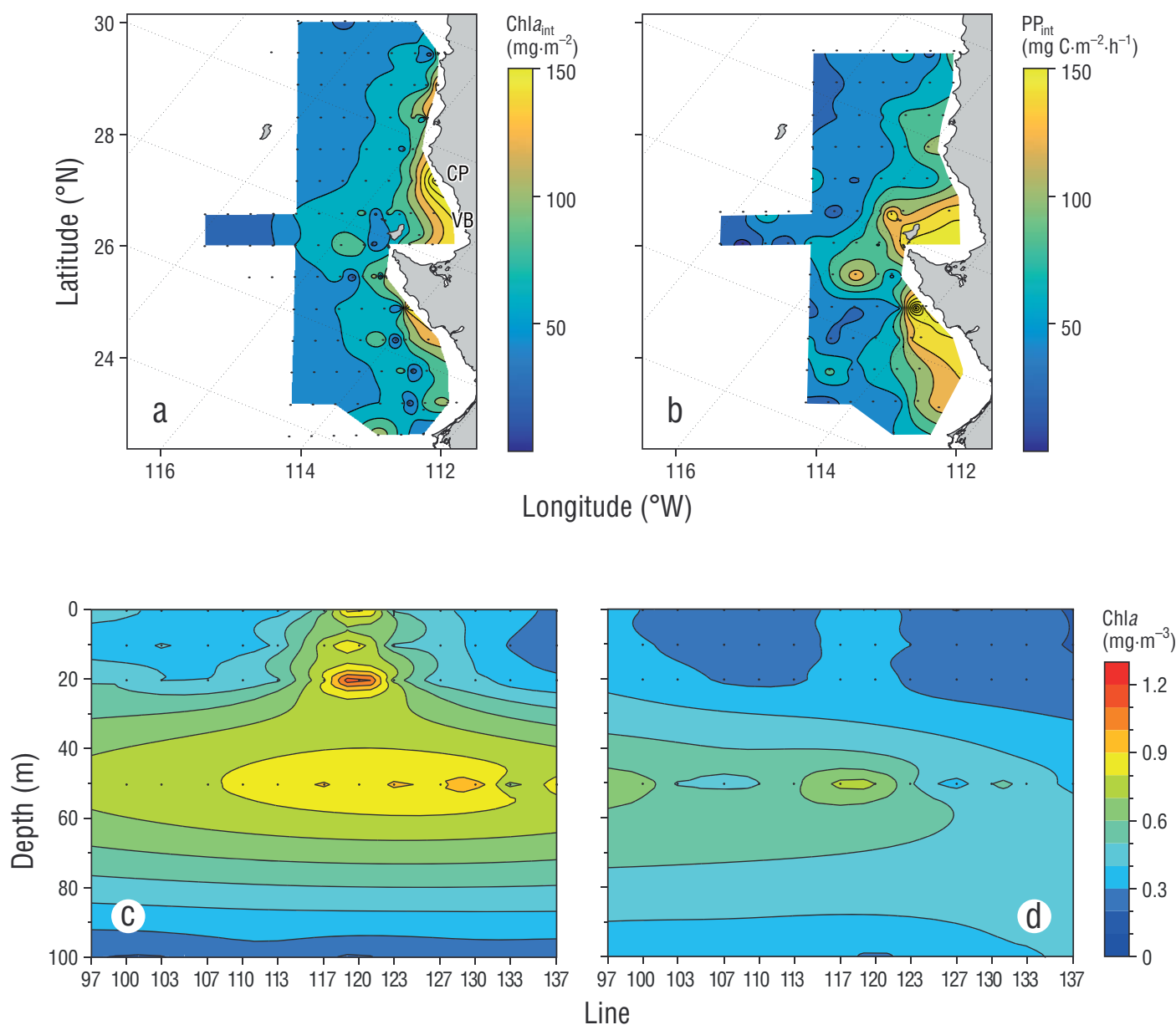

Figure 2. Mean horizontal distribution of in situ integrated chlorophyll $a\left(\mathrm{Chl}_{\mathrm{int}}\right.$, a) and primary production $\left(\mathrm{PP}_{\mathrm{int}}, \mathbf{b}\right)$, and vertical mean conditions $(\mathbf{c}, \mathbf{d})$ for chlorophyll $a(\mathrm{Chl} a)$ from the surface to $100 \mathrm{~m}$ depth. The vertical distributions correspond to station 45 (E45, c) and station 60 (E60, d) of each IMECOCAL line. Geographic references are Vizcaíno Bay (VB) and Canoas Point (CP). Mean conditions were estimated with at least 2 samples per station.

Figura 2. Distribución horizontal promedio de la clorofila $a$ integrada in situ $\left(\mathrm{Chla}_{\mathrm{int}}\right.$, a) y la producción primaria integrada in situ $\left(\mathrm{PP}_{\mathrm{int}}\right.$, b), y condiciones verticales promedio $(\mathbf{c}, \mathbf{d})$ para la clorofila $a($ Chla) desde la superficie hasta $100 \mathrm{~m}$ de profundidad. La distribución vertical corresponde a la estación $45(\mathrm{E} 45$, c) y la estación 60 (E60,d) de cada línea del programa IMECOCAL. Las referencias geograficas son bahía de Vizcaíno (VB) y punta Canoas (CP). Los promedios fueron calculados con 2 muestras por estación como mínimo. 
observed to the south of Punta Eugenia $\left(28^{\circ} \mathrm{N}\right)$. In spring $\mathrm{PP}_{\text {int }}$ maxima $\left(\sim 150 \mathrm{mg} \mathrm{C} \cdot \mathrm{m}^{-2} \cdot \mathrm{h}^{-1}\right)$ occurred in the region to the north of Punta Eugenia. During summer, the highest $\mathrm{PP}_{\text {int }}$ values $\left(\sim 150 \mathrm{mg} \mathrm{C} \cdot \mathrm{m}^{-2} \cdot \mathrm{h}^{-1}\right)$ occurred in Vizcaíno bay, whereas in the region to the south and off Punta Eugenia the highest values occurred during autumn.

\section{Phytoplankton biomass and production anomalies (1997-2016)}

Notable interannual variations of phytoplankton biomass and production integrated in the water column were observed throughout the 20 years of IMECOCAL surveys. Phytoplankton biomass decreased during various periods: El Niño in 1998, the subarctic water intrusion from 2002 to 2007, the Blob, and the 2015-2016 El Niño. The largest declines in Chla concentrations were observed during the subarctic water intrusion (approximately $-30 \mathrm{mg} \cdot \mathrm{m}^{-2}$ ) and El Niño events (approximately $-20 \mathrm{mg} \cdot \mathrm{m}^{-2}$ ). In contrast, the 1999 and 2008-2010 La Niña events and the intensified upwelling during 2002 increased phytoplankton biomass by up to $90 \mathrm{mg} \cdot \mathrm{m}^{-2}$ (Fig. 5a). Unlike biomass, phytoplankton de El Niño/Oscilación del Sur. Los mayores declives de la $\mathrm{PP}_{\text {int }}$ ocurrieron enseguida del evento de El Niño de 1998 (se redujo en $30 \mathrm{mg} \mathrm{C} \cdot \mathrm{m}^{-2} \cdot \mathrm{h}^{-1}$ ) y en la mayor parte del periodo de la intrusión de agua subártica (de hasta $-40 \mathrm{mg} \mathrm{C} \cdot \mathrm{m}^{-2} \cdot \mathrm{h}^{-1}$ menos que el promedio).

\section{Discusión}

Las condiciones promedio y la variación estacional de Cla, $\mathrm{Cla}_{\text {int }} \mathrm{y} \mathrm{PP}_{\text {int }}$ en la región frente a Baja California fueron caracterizadas en el estudio realizado por Gaxiola-Castro et al. (2010) con 11 años de mediciones del programa IMECOCAL. En este trabajo, nuestro objetivo fue, además de refinar tales promedios con 20 años de mediciones in situ, centrarnos en analizar la productividad horizontal a través de las distribuciones de la producción y la biomasa del fitoplancton integradas en la columna de agua, y la distribución vertical de Cla en 2 transectos representativos frente a Baja California. Además, analizamos el efecto de diferentes eventos ocurridos a gran escala durante 2010-2016 sobre la productividad. Debido a la importancia del fitoplancton como principal componente de la cadena trófica marina, este tipo de estudios se vuelven

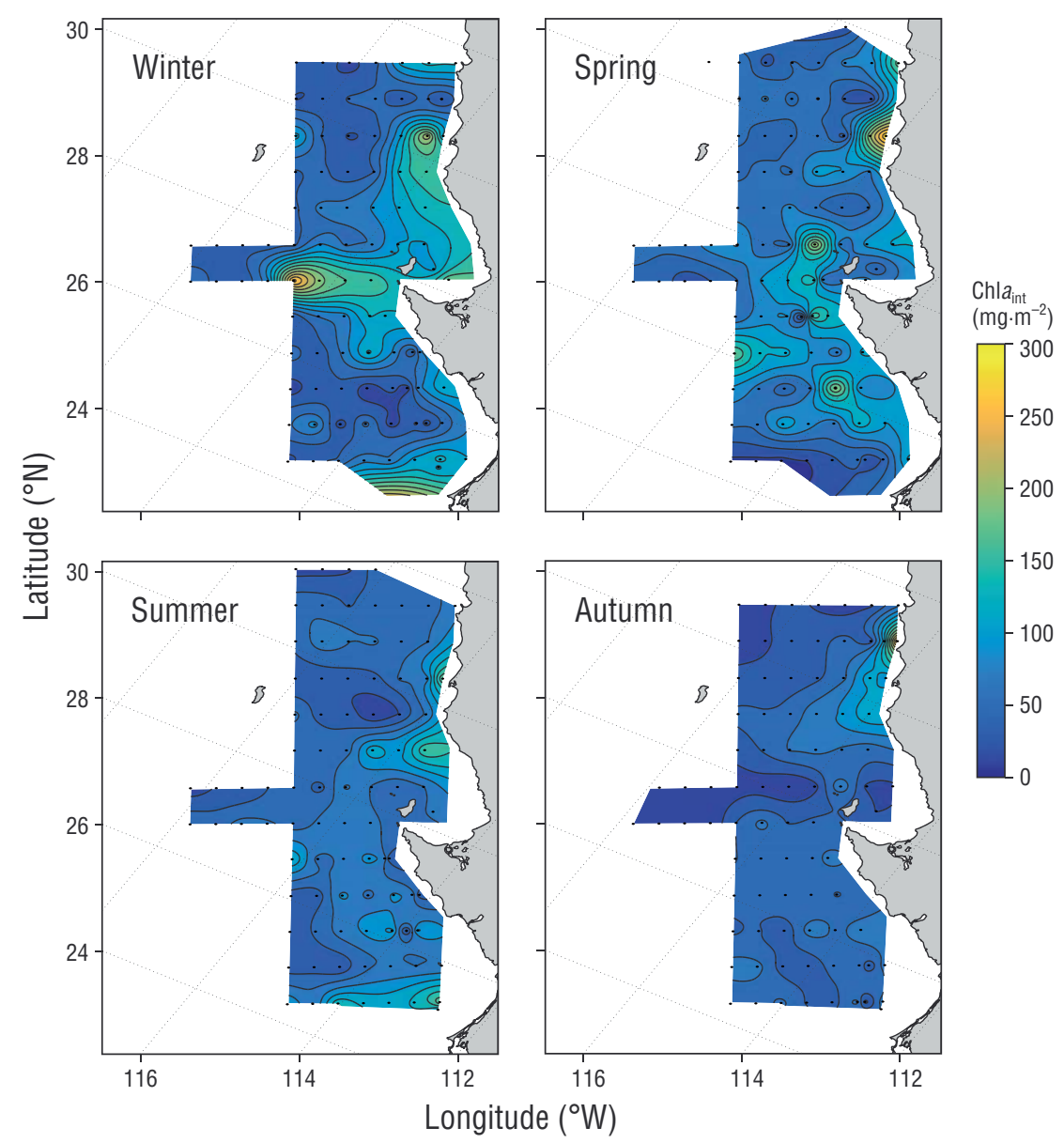

Figure 3. Seasonal variability of integrated chlorophyll $a\left(\mathrm{Chl}_{\text {int }}\right)$ in winter, spring, summer, and autumn.

Figura 3. Variabilidad estacional de la clorofila $a$ integrada $\left(C h l a_{\text {int }}\right)$ en invierno, primavera, verano y otoño. 
production (Fig. 5b) did not exhibit clear responses to interannual events. However, noticeable periods of $\mathrm{PP}_{\text {int }}$ increase were observed during the 1999 and 2008 La Niña events, in late 2014 during the transition period between the Blob and the 2015-2016 El Niño event, and in late 2016 during the ENSO neutral period. The largest $\mathrm{PP}_{\text {int }}$ decrease occurred immediately after the 1998 El Niño event $\left(-30 \mathrm{mg} \mathrm{C} \cdot \mathrm{m}^{-2} \cdot \mathrm{h}^{-1}\right)$ and during most of the period of subarctic water intrusion (up to $-40 \mathrm{mg} \mathrm{C} \cdot \mathrm{m}^{-2} \cdot \mathrm{h}^{-1}$ below the average).

\section{Discussion}

Gaxiola-Castro et al. (2010) used measurements from 11 years of surveys by the IMECOCAL program to characterize average conditions and the seasonal variability of Chla, $\mathrm{Chl}_{\text {int }}$, and $\mathrm{PP}_{\text {int }}$ in the region off Baja California. In the present study, in addition to refining these averages using in situ measurements from 20 years, our aim was to analyze horizontal productivity using phytoplankton production and biomass integrated in the water column, and the vertical distribution of Chla in 2 representative transects off Baja California. We also analyze the effect of the different large-scale events that relevantes para entender la variación regional en niveles tróficos superiores.

Se observó alta productividad en la zona costera de bahía de Vizcaíno y en la zona oceánica frente a punta Eugenia $\left(28^{\circ} \mathrm{N}\right)$. La bahía de Vizcaíno ha sido caracterizada como un ambiente mesotrófico influenciado por el agua de la CC, por las surgencias costeras generadas frente a punta Canoas $\left(29^{\circ} \mathrm{N}\right)$ y por un remolino anticiclónico superficial que advecta el agua superficial fría y rica en nutrientes hacia el interior de la bahía (Martínez-Fuentes et al. 2016). Las condiciones subárticas conferidas por la CC, y el afloramiento de aguas ricas en nutrientes sustentan la alta productividad de esta región. Adicionalmente, fuera de la bahía, se ha observado la presencia de 2 estructuras ciclónicas separadas por punta Eugenia (Durazo 2015), las cuales favorecen la disponibilidad de nutrientes para el fitoplancton a través del levantamiento de las isopicnas en la zona oceánica. Por lo tanto, el área de bahía de Vizcaíno y alrededor de punta Eugenia conforma un ambiente físicamente dinámico, lo cual propicia la alta productividad de esta región observada en las distribuciones de $\mathrm{PP}_{\text {int }} \mathrm{y}$ Cla $a_{\text {int }}$ (Fig. 2).

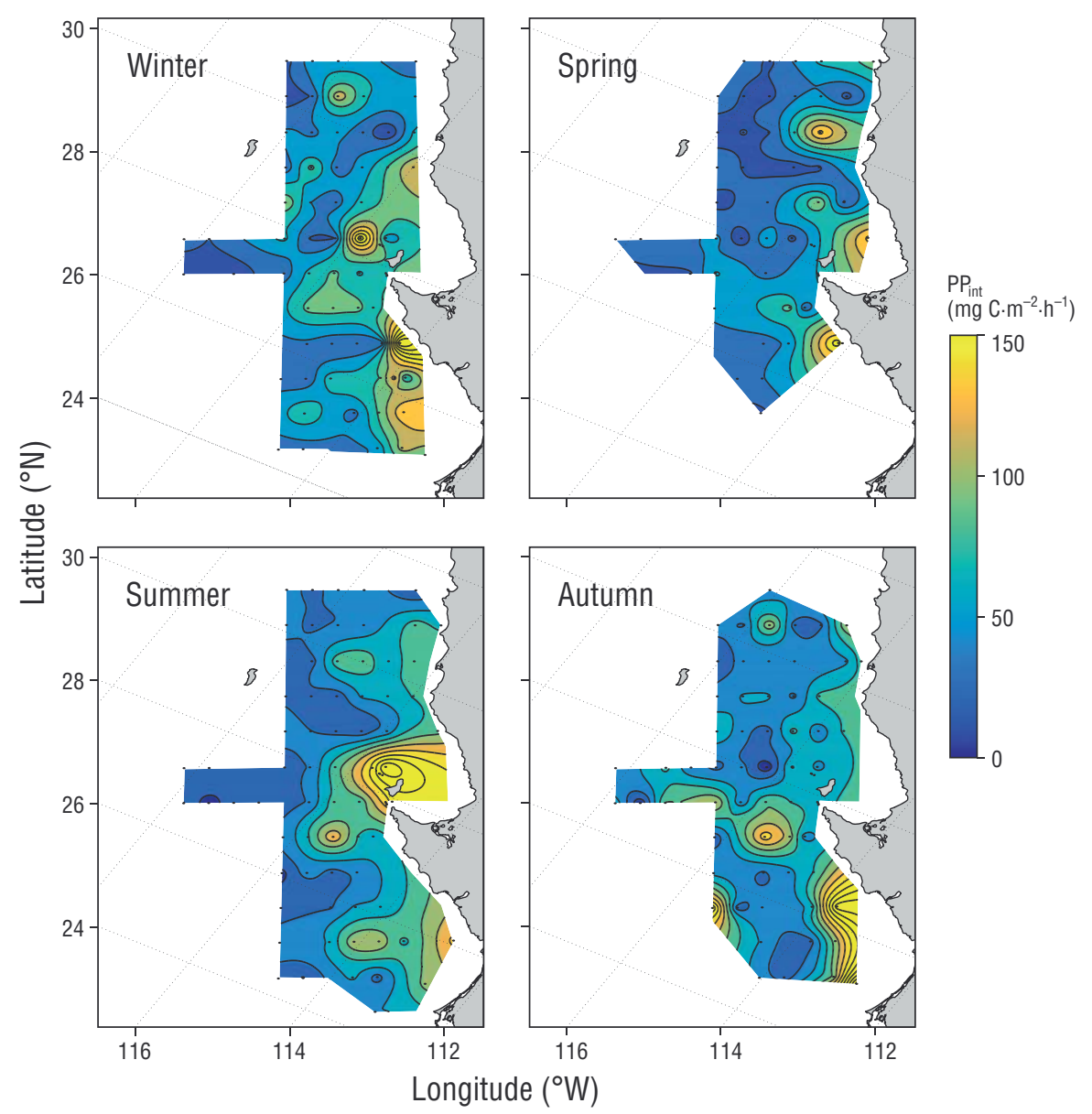

Figure 4. Seasonal variability of integrated primary production $\left(\mathrm{PP}_{\mathrm{int}}\right)$ in winter, spring, summer, and autumn.

Figura 4. Variabilidad estacional de la producción primaria integrada $\left(\mathrm{PP}_{\text {int }}\right)$ en invierno, primavera, verano y otoño. 
occurred in 2010-2016 on productivity. Since phytoplankton is the main component in the marine trophic food web, this type of study becomes relevant to understand the regional variations of upper trophic levels.

High productivity was observed in the Vizcaíno Bay coastal zone and in the oceanic zone off Punta Eugenia $\left(28^{\circ} \mathrm{N}\right)$. Vizcaíno Bay is characterized as a mesotrophic environment influenced by $\mathrm{CC}$ waters, coastal upwelling events off Punta Canoas $\left(29^{\circ} \mathrm{N}\right)$, and an anticyclonic surface eddy that advects the cool, nutrient-rich surface water into the bay (Martínez-Fuentes et al. 2016). The subarctic conditions conveyed by the $\mathrm{CC}$, and the upwelling of nutrient-rich waters sustain the high productivity in this region. In addition, outside the bay, there are 2 cyclonic structures separated by Punta Eugenia (Durazo 2015), which raise isopycnals in the oceanic zone and thus increase nutrient availability for phytoplankton. Therefore, the area covering Vizcaíno Bay and waters near Punta Eugenia is a physically dynamic environment that favors the regional high productivity observed in $\mathrm{PP}_{\text {int }}$ and $\mathrm{Chl} a_{\text {int }}$ distributions (Fig. 2).

Water column productivity in the oceanic zone was divided into 2 regions, a mesotrophic one covering the northernmost lines and an oligotrophic one covering the southernmost lines, with a marked maximum at station 45 of line 117 . These 2 productivity regions are a result of the influence of subarctic water transported by the $\mathrm{CC}$ throughout the year in the area north of Punta Eugenia (Durazo 2015), and of the input
La productividad de la columna de agua en la zona oceánica se dividió en 2 regiones, una mesotrófica en las líneas más norteñas y otra oligotrófica en las líneas más sureñas, con un máximo marcado en la estación 45 de la línea 117. Esta división en 2 regiones de productividad se genera por la influencia durante todo el año del agua subártica acarreada por la $\mathrm{CC}$ en la región al norte de punta Eugenia (Durazo 2015), sumado al aporte de aguas ricas en nutrientes proveniente de la boca sur de bahía de Vizcaíno y a las surgencias costeras (Martínez-Fuentes et al. 2016). En contraste, el área al sur de punta Eugenia está caracterizada por la influencia estacional de aguas cálidas de origen tropical y subtropical (Durazo 2015). Adicionalmente, el giro ciclónico semipermanente frente a punta Eugenia podría ser la causa del punto de alta productividad generado en esta área, visualizado en el máximo superficial de clorofila en la estación 45 de la línea 117 y en la distribución media de $\mathrm{Cl}_{\text {int }}$ y $\mathrm{PP}_{\text {int }}$. Es probable que la alta productividad observada frente a punta Eugenia sea producto de afloramientos de agua producidos por un efecto de punta (Largier et al. 2006). Por otro lado, los resultados de separación provincial por productividad son consistentes con la diferenciación de 2 regiones propuesta por Durazo (2015), una de carácter subártico al norte de punta Eugenia y otra al sur influenciada por aguas cálidas.

En la zona costera, los máximos de la biomasa de fitoplancton, reflejada en la $\mathrm{Cl}_{\text {int }}$ en la columna de agua, se presentaron en primavera, mientras que los mínimos ocurrieron en
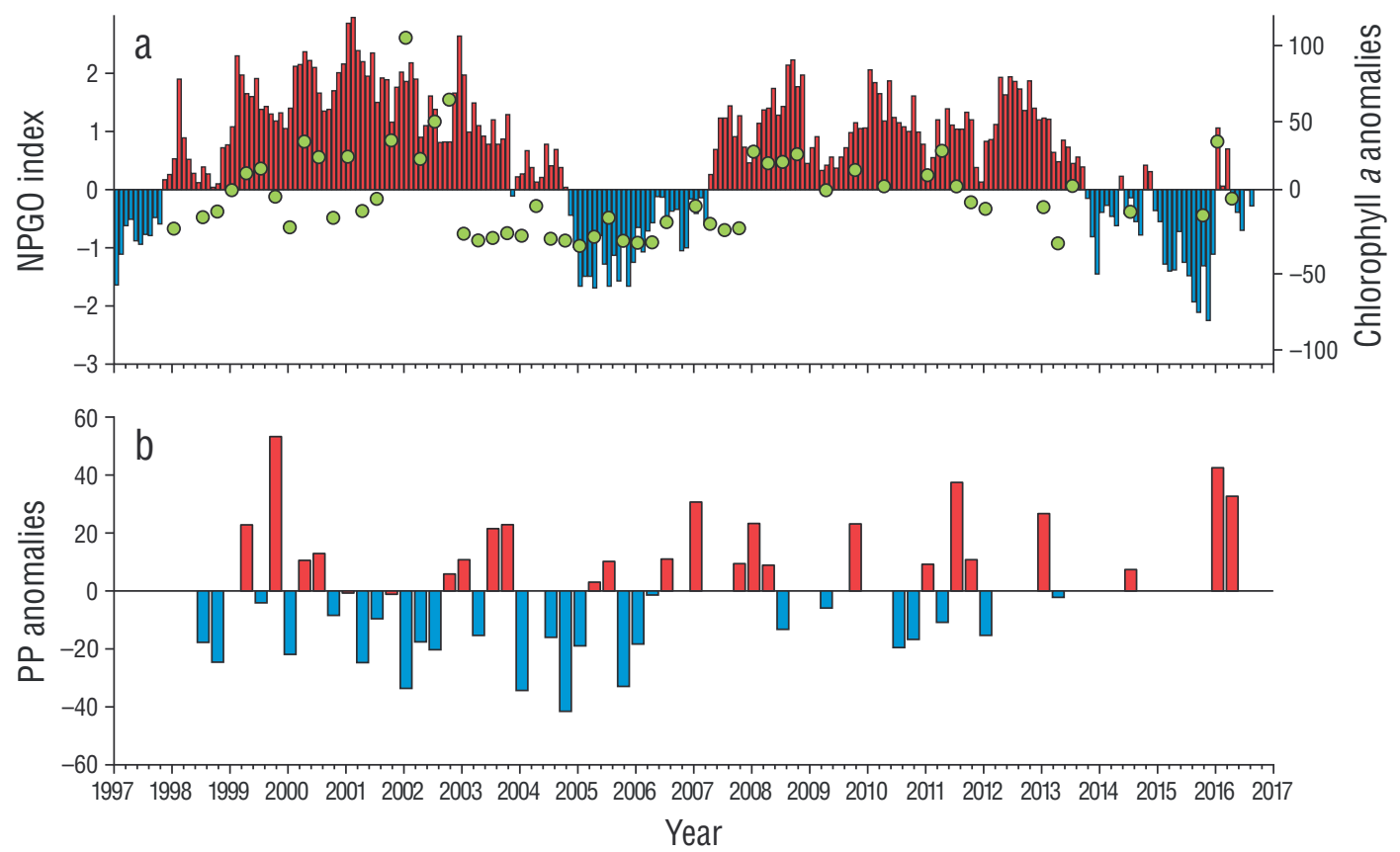

Figure 5. Time series anomalies for (a) integrated chlorophyll $a\left(\mathrm{mg} \cdot \mathrm{m}^{-2}\right.$, green circles) and the North Pacific Gyre Oscillation index (NPGO, bars), and (b) integrated primary production (PP anomalies, $\mathrm{mg} \mathrm{C} \cdot \mathrm{m}^{-2} \cdot \mathrm{h}^{-1}$ ). Red bars denote positive anomalies and blue bars negative anomalies.

Figura 5. Series de tiempo de las anomalías para (a) la clorofila $a$ integrada ( $\mathrm{mg} \cdot \mathrm{m}^{-2}$, círculos verdes) y el índice de la Oscilación del Giro de Pacifico Norte (NPGO, barras) y (b) la producción primaria integrada (anomalías de PP, $\mathrm{mg} \mathrm{C} \cdot \mathrm{m}^{-2} \cdot \mathrm{h}^{-1}$ ). Las barras rojas indican anomalías positivas y las barras azules indican anomalías negativas. 
De la Cruz-Orozco et al.: Phytoplankton biomass and production off Baja California

of nutrient-rich waters from the southern mouth of Vizcaíno Bay and coastal upwelling (Martínez-Fuentes et al. 2016). In contrast, the area to the south of Punta Eugenia is characterized by the seasonal influence of warm waters of tropical and subtropical origin (Durazo 2015). Furthermore, the semipermanent cyclonic gyre off Punta Eugenia could have favored the high productivity site found in this area, which can be observed by the surface chlorophyll maximum at station 45 of line 117 and in the $\mathrm{Chla}_{\text {int }}$ and $\mathrm{PP}_{\text {int }}$ average distribution. The high productivity observed off Punta Eugenia could be the result of upwelling waters produced by the effect of the headland (Largier et al. 2006). On the other hand, the regional division into productivity areas is consistent with the 2 regions proposed by Durazo (2015), one with subarctic characteristics to the north of Punta Eugenia and another one with warm water influence to the south.

In the coastal zone, phytoplankton biomass maxima, as seen by Chla $a_{\text {int }}$ in the water cloumn, occurred in spring and minima occurred in autumn. The observed maxima took place during the period of most intense coastal upwelling in the IMECOCAL region (Durazo 2015). Wind stress along the coast prompts upwelling of nutrient-rich subsurface waters, which favors phytoplankton growth and biomass increase. During autumn upwelling weakens and the influence of warm waters of subtropical and tropical origin to the north of Punta Eugenia causes biomass to decline because the nutricline deepens and the input of nutrients to the euphotic zone decreases. These results concur with the results obtained by Gaxiola-Castro et al. (2010) with 10 years of phytoplankton biomass and production data analysis for the IMECOCAL area. Nevertheless, the seasonal analysis of $\mathrm{Chl} a_{\text {int }}$ and $\mathrm{PP}_{\text {int }}$ spatial distributions allowed us to identify high productivity zones and their association with ocean circulation processes.

The seasonal variability of phytoplankton production in the water column showed a different pattern from that observed for biomass. The highest $\mathrm{PP}_{\text {int }}$ values occurred during spring and summer to the north of Punta Eugenia, whereas to the south of this location the highest values occurred during winter and autumn. Furthermore, during summer and autumn, the cyclonic gyre off Punta Eugenia affected this zone and increased productivity. During spring and summer, the turbulent, nutrient-rich environment originated by coastal upwelling, mainly to the north of Punta Eugenia (Durazo 2015, Martínez-Fuentes et al. 2016), favors the presence of larger phytoplankton cells $(20-200 \mu \mathrm{m})$ such as diatoms (Hinz et al. 2012). On the contrary, during autumn and winter the warm and oligotrophic environment prompted by the intrusion of tropical and subtropical waters in the area to the south of Punta Eugenia provides ideal conditions for the presence of smaller phytoplankton cells (e.g., Picoplankton 0.2-2.0 $\mu \mathrm{m}$ and nanoplankton 2.0-20 $\mu \mathrm{m}$ ) (Barber and Hiscock 2006). The differences in phytoplankton cell sizes have been associated with the magnitudes of 2 photosynthetic parameters: maximum utilization coefficient at low light conditions $\left(\alpha^{\mathrm{B}}\right)$ and maximum photosynthetic rate $\left(P_{m}^{B}\right)$ (Coté and Platt 1983). A $P_{m}^{B}$ increase could be associated with otoño. Los máximos observados corresponden a la época de las surgencias costeras más intensas en la región IMECOCAL (Durazo 2015). El efecto del esfuerzo del viento que sopla a lo largo de la costa propicia el afloramiento de aguas subsuperficiales ricas en nutrientes, lo cual promueve el crecimiento y el incremento de biomasa del fitoplancton. En otoño, las surgencias se debilitan y la influencia de agua cálida de origen tropical-subtropical en esta época en el área al norte de punta Eugenia produce la disminución de la biomasa por el desabastecimiento de nutrientes en la zona eufótica causada por la profundización de la nutriclina. Estos resultados concuerdan con lo reportado por Gaxiola-Castro et al. (2010) con 10 años de datos de biomasa y producción de fitoplancton en el área IMECOCAL. Sin embargo, el análisis estacional de la distribución espacial de $\mathrm{Cl}_{\text {int }}$ y $\mathrm{PP}_{\text {int }}$ permitió diferenciar zonas de alta productividad y su relación con procesos de circulación oceánica.

La variación estacional de la producción del fitoplancton en la columna de agua tuvo un patrón diferente al observado para la biomasa. Los mayores valores de $\mathrm{PP}_{\text {int }}$ ocurrieron en primavera y verano al norte de punta Eugenia, mientras que al sur de esta localidad los mayores valores se presentaron en invierno y otoño. Adicionalmente, durante el verano y el otoño se observó el efecto del giro ciclónico frente de punta Eugenia como un aumento en la productividad de esta zona. Durante la primavera y el verano, el ambiente turbulento y rico en nutrientes propiciado por las surgencias costeras, principalmente al norte de punta Eugenia (Durazo 2015, Martínez-Fuentes et al. 2016), favorece el establecimiento de fitoplancton de mayor tamaño $(20-200 \mu \mathrm{m})$ como las diatomeas (Hinz et al. 2012). En contraste, durante el otoño y el invierno el ambiente cálido y oligotrófico generado por la entrada de agua de origen tropical-subtropical en el área al sur de punta Eugenia establece condiciones idóneas para la presencia de organismos fitoplanctónicos de menor tamaño (e.g., picoplancton de 0.2 a $2 \mu \mathrm{m}$ y nanoplancton de 2.0 a $20 \mu \mathrm{m})$ (Barber y Hiscock 2006). Anteriormente, se han relacionado las diferencias en el tamaño del fitoplancton con las magnitudes de 2 parámetros fotosintéticos: el coeficiente de máxima utilización a bajas condiciones de luz $\left(\alpha^{\mathrm{B}}\right)$ y la tasa fotosintética máxima $\left(P_{m}^{B}\right)$ (Coté y Platt 1983). El incremento de $P_{m}^{B}$ podría estar relacionado con la fotoaclimatación a altas irradiancias de pequeñas células de fitoplancton, como cianofitas y proclorofitas, tal y como ocurre en ambientes con alta estratificación donde la termoclina y la capa de mezcla son someras. Geider et al. (1993) observaron cambios de $P_{m}^{*}$ entre células de fitoplancton de diferente tamaño, donde las diatomeas grandes tuvieron bajas $P_{m}^{*}$ a la misma irradiancia de crecimiento. Similarmente, GómezOcampo et al. (2017a) observaron $P_{m}^{B}$ más altas en el área IMECOCAL en épocas de alta estratificación. Lo anterior explicaría los máximos de $\mathrm{PP}_{\text {int }}$ al sur del área IMECOCAL durante el otoño y el invierno (Fig. 4d, 4a). En concreto, la circulación oceánica del área IMECOCAL caracterizada por las masas de agua presentes condiciona el hábitat para 
the photoacclimation of small phytoplankton cells (e.g., cyanophytes and prochlorophytes) to high irradiances, as has occurred in highly stratified environments where the thermocline and the mixed layer are shallow. Geider et al. (1993) observed $P_{m}^{*}$ changes between different phytoplankton cell sizes and reported that large diatoms showed low $P_{m}^{*}$ at the same growth irradiance. Moreover, Gómez-Ocampo et al. (2017a) observed higher $P_{m}^{B}$ in the IMECOCAL area during high stratification periods. This could explain $\mathrm{PP}_{\text {int }}$ maxima to the south of the IMECOCAL area during autumn and winter (Fig. 4d, a). On the whole, oceanic circulation in the IMECOCAL area, which is characterized by the water masses in it, determines phytoplankton habitat and favors the presence of various phytoplankton cell sizes with different photosynthetic efficiencies.

The largest declines in phytoplankton biomass and production integrated in the water column occurred during the subarctic water intrusions and El Niño events. In the 20-year time frame, the period of subarctic water intrusion was always associated with the largest productivity decline off Baja California. This could be due to the high water-column stratification and pycnocline deepening that occurred during this period, particularly in the oceanic zone (Gómez-Ocampo et al. 2017a). Both conditions limited nutrient input in the euphotic zone, and this was reflected in the reduction of phytoplankton biomass and primary production rates. However, the effect of zooplankton grazing on low chlorophyll values should not be discarded because zooplankton volumes increased from 2003 to 2013 (Lavaniegos et al. 2015). The other period with decreased productivity occurred during the Blob and El Niño 2015-2016, and it was consistent with that reported by Gómez-Ocampo et al. (2017b), who analyzed satellite-derived chlorophyll data and modeled PP data for the southern end of the CC. Unlike in the period of subarctic water intrusion, during this period stratification was high but the pycnocline was shallower. This could explain the less drastic, yet high, productivity decline during this period. Moreover, zooplankton volume decreased drastically during the Blob; hence, grazing pressure was discarded as an influential factor in the decline of chlorophyll concentrations (McClatchie et al. 2016).

In order to associate atmospheric and oceanic processes with productivity anomalies, correlations between the interannual indexes (NPGO, PDO, and MEI) and the time series of Chl $a_{\text {int }}$ and $\mathrm{PP}_{\text {int }}$ anomalies were calculated for the analyzed period. Correlations with MEI and PDO, though significant, were low $(<0.3$, results not shown). On the other hand, the highest correlation ( $\sim 0.5,95 \%$ confidence interval) occurred between NPGO and the time series of Chla $a_{\text {int }}$ anomalies. The high correlation in the area was previously reported for $\mathrm{Chl} a_{\text {int }}$ anomalies (Gaxiola-Castro et al. 2008) and zooplankton (Lavaniegos et al. 2015). Negative NPGO values are associated with the weakening of the $\mathrm{CC}$ and strengthening of the Aleutian Low (weakening winds along the coast), which altogether reduce the input of cool, subarctic waters, weak coastal upwelling (Chhak and Di Lorenzo 2007, Di Lorenzo et al. 2008), and consequently nutrient input into the euphotic el fitoplancton, lo cual da lugar al establecimiento de fitoplancton de diversos tamaños con eficiencias fotosintéticas diferentes.

Los mayores declives en la biomasa y la producción del fitoplancton integradas en la columna de agua ocurrieron durante la intrusión de agua subártica y los periodos El Niño. En los 20 años analizados, el tiempo durante la intrusión de agua subártica siguió siendo el periodo en cual se registró la mayor reducción en la productividad frente a Baja California. Lo anterior puede deberse a que durante este periodo se presentó una alta estratificación de la columna de agua y profundización de la picnoclina, particularmente en la zona oceánica (Gómez-Ocampo et al. 2017a). Ambas condiciones suprimen el ingreso de nutrientes hacia la zona eufótica, lo cual se ve reflejado en la reducción de las tasas de producción primaria y biomasa del fitoplancton. No se debe descartar un posible efecto del pastoreo de zooplancton en los bajos valores de clorofila, ya que de 2003 a 2013 se observó un incremento en los volúmenes de zooplancton (Lavaniegos et al. 2015). El siguiente periodo en el que la productividad disminuyó ocurrió durante el Blob y El Niño de 2015-2016 y fue también consistente con lo reportado por Gómez-Ocampo et al. (2017b), quienes analizaron datos de clorofila de satélite y producción primaria modelada para la zona sur de la CC. A diferencia del periodo de intrusión de agua subártica, en este periodo se registró alta estratificación pero con picnoclina más superficial. Esto explicaría la reducción menos drástica, aunque alta, de la productividad en este periodo. Por otro lado, el volumen de zooplancton disminuyó drásticamente durante el Blob, por lo que se descarta una presión por pastoreo como un factor influyente de las bajas concentraciones de clorofila (McClatchie et al. 2016).

Con el fin de relacionar los procesos atmosféricos y oceánicos con las anomalías en la productividad, se calcularon las correlaciones de los índices interanuales (NPGO, PDO y MEI) con las series de anomalías de $\mathrm{Cla}_{\text {int }}$ y $\mathrm{PP}_{\text {int }}$ para el periodo analizado. Las correlaciones con el MEI y el PDO, aunque fueron significativas, resultaron bajas $(<0.3$, resultados no mostrados). Por otra parte, la correlación más alta ( $0.5,95 \%$ nivel de confianza) se presentó entre el NPGO y la serie de tiempo de las anomalías de la $\mathrm{Cl}_{\text {int }}$. Esta correlación alta ya había sido reportada anteriormente en la zona para las anomalías de $\mathrm{Cla}_{\text {int }}$ (Gaxiola-Castro et al. 2008) y el zooplancton (Lavaniegos et al. 2015). Los valores negativos del índice del NPGO están relacionados con el debilitamiento de la $\mathrm{CC}$ y el fortalecimiento del bajo aleutiano (debilitamiento de vientos a lo largo de la costa), lo cual reduce el aporte de aguas frías de origen subártico, el debilitamiento de las surgencias costeras (Chhak y Di Lorenzo 2007, Di Lorenzo et al. 2008) y, consecuentemente, el transporte de nutrientes a la zona eufótica. Lavaniegos et al. (2015) observaron, para 4 regiones frente a Baja California, correlaciones significativas entre las anomalías de salinidad superficial del mar y el NPGO, con presencia de aguas 
De la Cruz-Orozco et al.: Phytoplankton biomass and production off Baja California

zone. Lavaniegos et al. (2015) observed significant correlations between sea surface salinity anomalies and NPGO in 4 regions off Baja California, with the presence of more saline waters during the positive NPGO phases, probably due to the strengthening of coastal upwelling. During the 2 periods of highly reduced productivity, the phase of the NPGO was negative (Fig. 5). This correlation indicated that atmospheric processes originating in the North Pacific modulate productivity off the Baja California Peninsula.

\section{ACKNOWLEDGMENTS}

This work is dedicated to the memory of Gilberto Gaxiola-Castro $^{\dagger}$, who was a teacher, a friend, and founder of the IMECOCAL program. Financial support was provided by the National Council for Science and Technology (Mexico; projects no. 23804, 23947, 255602, 254745) and CICESE (project no. 625114). We thank the crew members who participated in the oceanographic cruises and the vessel department for all their support in extracting the biological samples used for this project.

English translation by Claudia Michel-Villalobos.

\section{REFERENCIAS}

Barber RT, Hiscock MR. 2006. A rising tide lifts all phytoplankton: Growth response of other phytoplankton taxa in diatomdominated blooms. Global Biogeochem. Cycles 20(4): 1-12. https://doi.org/10.1029/2006gb002726

Bograd SJ, Lynn RJ. 2003. Anomalous subarctic influence in the southern California Current during 2002. Geophys. Res. Lett. 30(15): 8020. https://doi.org/10.1029/2003gl017446

Chhak K, Di Lorenzo E. 2007. Decadal variations in the California Current upwelling cells. Geophys. Res. Lett. 34(14): 1-6. https://doi.org/10.1029/2007g1030203

Côté B, Platt T. 1983. Day-to-day variations in the spring-summer photosynthetic parameters of coastal marine phytoplankton. Limnol. Oceanogr. 28(2): 320-344. https://doi.org/10.4319/lo.1983.28.2.0320

Di Lorenzo E, Schneider N, Cobb KM, Franks PJS, Chhak K, Miller AJ, Mcwilliams JC, et al. 2008. North Pacific Gyre Oscillation links ocean climate and ecosystem change. Geophys. Res. Lett. 35(8): L08607. https://doi.org/10.1029/2007g1032838

Durazo R. 2009. Climate and upper ocean variability off Baja California, Mexico: 1997-2008. Prog. in Oceanogr. 83(1-4): 361-368.

https://doi.org/10.1016/j.pocean.2009.07.043

Durazo R. 2015. Seasonality of the transitional region of the California Current System off Baja California. J. Geophys. Res.: Oceans 120(2): 1173-1196. https://doi.org/10.1002/2014jc010405

Espinosa-Carreón TL, Gaxiola-Castro G, Beier E, Strub PT, Kurczyn JA. 2012. Effects of mesoscale processes on phytoplankton chlorophyll off Baja California. J. Geophys. Res.: Oceans 117(C4): 112. https://doi.org/10.1029/2011jc007604

Espinosa-Carreón TL, Gaxiola-Castro G, Durazo R, De la CruzOrozco ME, Norzagaray-Campos M, Solana-Arellano E. 2015. Influence of anomalous subarctic water intrusion on más salinas durante fases positivas del NPGO, probablemente debidas al fortalecimiento de las surgencias costeras. Durante los 2 periodos de reducción alta en la productividad, el NPGO se encontraba en fase negativa (Fig. 5). Esta correlación indica que los procesos atmosféricos originados en el Pacifico Norte modulan la productividad frente a la península de Baja California.

\section{Agradecimientos}

Dedicamos este artículo a la memoria de Gilberto Gaxiola ${ }^{\dagger}$, maestro, amigo y fundador del programa IMECOCAL. El apoyo financiero provino del Consejo Nacional de Ciencia y Tecnología (México; proyectos 23804, 23947, 255602, 254745) y del CICESE (proyecto 625114). Agradecemos al personal participante en los cruceros oceanográficos y al departamento de embarcaciones todo su apoyo para extraer del mar el preciado material biológico con que se realizó este proyecto.

phytoplankton production off Baja California. Cont. Shelf Res. 92: $108-121$. https://doi.org/10.1016/j.csr.2014.10.003

Espinosa-Carreon TL, Strub PT, Beier E, Ocampo-Torres F, Gaxiola-Castro G. 2004. Seasonal and interannual variability of satellite-derived chlorophyll pigment, surface height, and temperature off Baja California. J. Geophys. Res. 109(C3): $1-20$. https://doi.org/10.1029/2003jc002105

Gaxiola-Castro G, Cepeda-Morales J, Nájera-Martínez S, EspinosaCarreón TL, De la Cruz-Orozco, ME Sosa-Avalos R, AguirreHernández E, Cantú-Ontiveros J. 2010. Biomasa y producción del fitoplancton [Biomass and phytoplankton production]. In: Gaxiola-Castro G, Durazo R (eds.), Dinámica del Ecosistema Pelágico frente a Baja California, 1977-2007: Diez años de Investigaciones Mexicanas de la Corriente de California [Pelagic Ecosystem dynamics off Baja California, 1997-2007: Ten years of Mexican Research on the California Current]. Secretaría de Medio Ambiente y Recursos Naturales, Mexico, $504 \mathrm{pp}$.

Gaxiola-Castro G, Durazo R, Lavaniegos B, De La Cruz-Orozco ME, Millán-Núñez E, Soto-Mardones L, Cepeda-Morales J. 2008. Pelagic ecosystem response to interannual variability off Baja California $=$ Respuesta del ecosistema pelágico a la variabilidad interanual del océano frente a Baja California. Cienc. Mar. 34(2): 263-270. http://dx.doi.org/10.7773/cm.v34i2.1413

Geider RJ, Roche J, Greene RM, Olaizola M. 1993. Response of the photosynthetic apparatus of Phaeodactylum tricornutum (Bacillariophyceae) to nitrate, phosphate, or iron starvation. J. Phycol. 29(6): 755-766. https://doi.org/10.1111/j.0022-3646.1993.00755.x

Gómez-Ocampo E, Durazo R, Gaxiola-Castro G, De la CruzOrozco M, Sosa-Avalos R. 2017a. Effects of the interannual variability of water column stratification on phytoplankton production and biomass in the northern zone off Baja 
California $=$ Efectos de la variabilidad interanual de la estratificación de la columna de agua sobre la producción y biomasa del fitoplancton en la zona norte frente a Baja California. Cienc. Mar. 43(2):109-122. http://dx.doi.org/10.7773/cm.v43i2.2759

Gómez-Ocampo E, Gaxiola-Castro G, Durazo R, Beier E. 2017b. Effects of the 2013-2016 warm anomalies on the California Current phytoplankton. Deep-Sea Res. (II Top. Stud. Oceanogr.) $0-1$. https://doi.org/10.1016/j.dsr2.2017.01.005

Hickey BM. 1998. Coastal oceanography of western north America from the tip of Baja California to Vancouver Island. In: Robinson AR, Brink KH. (eds.), The Global Coastal OceanRegional Studies and Syntheses. Harvad university press, Cambridge, Masschusetts, $1090 \mathrm{pp}$.

Hinz DJ, Nielsdóttir MC, Korb RE, Whitehouse MJ, Poulton AJ, Moore CM, Achterberg EP, Bibby TS. 2012. Responses of microplankton community structure to iron addition in the Scotia Sea. Deep-Sea Res. (II Top. Stud. Oceanogr.) 59-60: 36-46. https://doi.org/10.1016/j.dsr2.2011.08.006

Holm-Hansen O, Lorenzen CJ, Holmes RW, Strickland JDH. 1965. Fluorometric Determination of Chlorophyll. ICES J. Mar. Sci. 30(1): 3-15. https://doi.org/10.1093/icesjms/30.1.3

Kahru M, Mitchell BG. 2002. Influence of the El Niño-La Niña cycle on satellite-derived primary production in the California Current. Geophys. Res. Lett. 29(17): 27-1-27-4. https://doi.org/10.1029/2002gl014963

Largier JL, Lawrence CA, Roughan M, Kaplan DM, Dever EP, Dorman CE, Kudela RM, et al. 2006. WEST: A northern California study of the role of wind-driven transport in the productivity of coastal plankton communities. Deep-Sea Res. (II Top. Stud. Oceanogr.) 53(25--26): 2833-2849.

https://doi.org/10.1016/j.dsr2.2006.08.018
Lavaniegos BE, Molina-González O, Murcia-Riaño M. 2015. Zooplankton functional groups from the California Current and climate variability during 1997-2013. CICIMAR Oceánides 30(1): 45-62.

Lavaniegos BE, Ohman MD. 2003. Long-term changes in pelagic tunicates of the California Current. Deep-Sea Res. (II Top. Stud. Oceanogr.) 50(14-16): 2473-2498. https://doi.org/10.1016/s0967-0645(03)00132-2

Mantua NJ, Hare SR, Zhang Y, Wallace JM, Francis RC. 1997. A Pacific Interdecadal Climate Oscillation with Impacts on Salmon Production. Bull. Am. Meteorol.Soc. 78(6): 1069-1079. https://doi.org/10.1175/1520-0477(1997)078<1069:apicow>2.0. co;2

Martínez-Fuentes LM, Gaxiola-Castro G, Gómez-Ocampo E, Kahru M. 2016. Effects of interannual events (1997-2012) on the hydrography and phytoplankton biomass of Sebastián Vizcaíno Bay $=$ Efectos de eventos interanuales (1997-2012) sobre la variabilidad hidrográfica y biomasa del fitoplancton en bahía Sebastián Vizcaíno. Cienc. Mar. 42(2): 81-97. https://doi.org/10.7773/cm.v42i2.2626

McClatchie S, Goericke R, Leising A, Auth TD, Bjorkstedt E, Robertson RR, Brodeur RD, Du X, Daly EA, Morgan CA, et al. 2016. State of the California Current 2015-16: Comparisons with the 1997-98 El Niño. CalCOFI Rep. (57):5-61.

Roden GI. 1971. Aspects of the transition zone in the Northeastern Pacific. J. Geophys. Res. 76(15): 3462-3475. https://doi.org/10.1029/jc076i015p03462

Steeman-Nielsen E. 1952. The use of radio-active carbon $\left({ }^{14} \mathrm{C}\right)$ for measuring organic production in the sea. ICES J. Mar. Scie. 18(2): $117-140$. https://doi.org/10.1093/icesjms/18.2.117

Yentsch CS, Menzel DW. 1963. A method for the determination of phytoplankton chlorophyll and phaeophytin by fluorescence. Deep Sea Res. Oceanogr. Abstr. 10(3): 221-231. https://doi.org/10.1016/0011-7471(63)90358-9

Received April 2017, Accepted October 2017. 